\title{
Advanced Glycation End Products (AGEs) Antibody Protects Against AGEs-Induced Apoptosis and NF-кB p65 Subunit Overexpression in Rat Glomerular Culture
}

\author{
Oktavia Rahayu Adianingsih ${ }^{1 *}$, Diana Lyrawati ${ }^{1}$, Nur Samsu $^{2}$
}

\author{
${ }^{1}$ Laboratory of Pharmacy, Faculty of Medicine, Brawijaya University, Malang, Indonesia
}

${ }^{2}$ Division of Nephrology and Hypertension, Department of Internal Medicine, dr. Saiful Anwar Public Hospital, Malang, Indonesia

\section{ABSTRACT}

Advanced glycation end products (AGEs) have been thought to be a major cause of diabetic nephropathy (DN). The mechanisms underlying the involvement of AGEs antibody in diabetic nephropathy are not fully understood. The present study was designed to investigate the protective effect of AGEs antibody on AGEs-induced glomerular damage. Isolated glomeruli were pre-incubated either with $10 \mu \mathrm{g} / \mathrm{mL}$ polyclonal anti-AGEs antibody (AGE-pAb) or monoclonal anti- $\mathrm{N}^{3}$-carboxymethyl-lysine antibody (CML-mAb) as a model of AGEs antibody to block interaction of AGEs with receptor for AGEs (RAGE) and incubated afterwards either with $100 \mu \mathrm{g} / \mathrm{mL}$ bovine serum albumin (BSA) or AGE-modified bovine serum albumin (AGE-BSA) for $48 \mathrm{~h}$. Annexin V/nephrin doublestaining was performed to determine apoptosis. Using immunofluorescence, we found that administration of AGE-BSA not only significantly increased glomerular cells apoptosis and nuclear factor kappa B (NF-kB) p65 expression, but also reduced expression of nephrin, an important structural and signal molecule of podocytes slit diaphragm. Blocking the interaction of AGE-RAGE with AGEs antibody significantly protected glomerular cells from AGEs-induced apoptosis and NF- $\mathrm{kB}$ p65 overexpression. We found that AGE-pAb conferred superior protective effect compared with CmL-mAb for the same reduction in apoptosis and NF- $\mathrm{kB}$ p65 expression. In sharp contrast, CmL-mAb led to preserve expression of podocytes nephrin better than AGE-pAb. These results demonstrate that the antibody against AGEs may be beneficial for preventing the glomerular damage in DN.

Keywords: Advanced glycation end products; antibody, apoptosis, diabetic nephropathy, $N^{e}$-(carboxymethyl)lysine

\section{INTRODUCTION}

Diabetes mellitus (DM) is a significant health problem with a worldwide mortality around 382 million people in 2013, and this number is expected to rise to 592 million by 2035 [1]. AGEs are a causative factor in diabetic vascular complication such as diabetic nephropathy (DN) which further lead to end-stage renal disease [2,3]. Accumulation of AGEs is present in all renal compartments in diabetic patient with three mechanisms of toxicity include interaction AGEs with receptor for AGE (RAGE), in situ glycation and tissue deposition [4]. Inhibition of AGEs is widely regarded as an implicit goal in clinical medicine for the treatment of DN [5]. So far, most studies have been focused on the potential of therapies that not only target

\footnotetext{
*Corresponding author:

Oktavia Rahayu Adianingsih

Laboratory of Pharmacy, Faculty of Medicine, Brawijaya University

Jalan Veteran, Malang, East Java 65145, Indonesia

E-mail: oktavia.rahayu@ub.ac.id
}

various pathways upregulated by AGEs, but also target AGEs itself by preventing AGE formation, breaking AGE-protein cross-linking, or neutralizing AGE [611]. In addition to the development of anti-AGE activity from natural product or synthetic compound, immunization of AGEs in diabetic mice has recently attached attention [12].

AGEs have antigenic properties that may exert an autoimmune response. Autoantibodies against AGEs are detected in serum of patients with DM and nonDM which may play a role in the macrophage uptake of AGEs-modified protein via AGEs-immune complexes (AGE-IC) formation [13,14]. Disruption of the balance between AGEs formation and AGEs elimination will lead to accumulation of AGEs [14,15]. How-

\section{How to cite:}

Adianingsih OR, Lyrawati D, Samsu N (2016) Advanced Glycation End Products (AGEs) Antibody Protects Against AGEsInduced Apoptosis and NF-kB p65 Subunit Overexpression in Rat Glomerular Culture. J. Trop. Life. Science 6 (3): 176 - 183. 
ever, our knowledge of the molecular mechanism governing role of AGE-Ab and AGE-IC in diabetic complication is still very limited. Thus, in this research, we aimed to demonstrate the role of AGE- $\mathrm{Ab}$ in prevent AGEs-induced glomerular damage.

\section{MATERIALS AND METHODS Ethics}

This research was approved by the Health Research Ethics Commission from Faculty of Medicine, Brawijaya University, Malang, Indonesia, with registration number 356B/EC/KEPK-S2/06/2015.

\section{Chemicals}

All chemicals were purchased from Sigma (St. Louis, MO) unless otherwise indicated. AGE-mAb was purchased from Circulex (Nagano, Japan). AGE-pAb was purchased from Abcam (Cambridge, England). BSA (fraction V) was purchased from Roche Diagnostic GmbH (Mannheim, Germany). AGE-BSA was purchased from BioVision (California, USA). For immunofluorescence assay, the following primary antibodies were used: rabbit polyclonal anti-NF-kB p65 (Bioss, Woburn, USA), goat monoclonal anti-nephrin (Santa Cruz Biotechnology, California, USA), rabbit polyclonal anti-nephrin (Bioss, Woburn, USA) and Annexin $\mathrm{V}$ conjugated fluorescein-isothiocyanate (FITC) (BioLegend, Fell, Germany). Secondary antibodies were used: goat anti-rabbit IgG-FITC, rabbit anti-goat IgGrhodamine and goat anti-rabbit IgG-rhodamine (Santa Cruz Biotechnology, California, USA). Fetal bovine serum (FBS) was purchased from Gibco (Grand Island, NY, USA).

\section{Rat glomerular isolation and culture}

Isolation and culture of glomeruli were done as described previously with some modifications [16-19]. Briefly, male Wistar rats at age 7 - 9 weeks were sacrificed by cervical dislocation and perfused with ice-cold Hank's balanced salt solution (HBSS) through the heart. The kidneys were perfused with ice-cold HBSS and dissected into small pieces $\left(1-2 \mathrm{~mm}^{3}\right.$ cubes $)$ with a surgical blade in ice-cold HBSS. Next, the tissues were digested in collagenase solution containing 1 $\mathrm{mg} / \mathrm{mL}$ collagenase $\mathrm{A}$ in $\mathrm{HBSS}$ at $37^{\circ} \mathrm{C}$ for 1 hour with gentle agitation. The collagenase-digested tissues were gently pressed through a $100 \mu \mathrm{m}$ cell strainer (BD Bioscience, Bedford, USA), followed by flushing with 5 $\mathrm{mL}$ of ice-cold sterile HBSS. The cell suspension was then centrifuged at $200 \times \mathrm{g}$ for 5 minutes. The supernatant was discarded and the cell pellet was resus- pended in $2 \mathrm{~mL}$ of ice-cold HBSS. Glomeruli were isolated manually with micro-hematocrit tube and transferred at least two times into another dish to remove any remaining debris which included when isolation process. All procedure were performed on ice, except for the collagenase digestion. Isolated glomeruli were cultured on type I collagen-coated glass-bottom culture dishes (MatTek Corporation, Ashland, MA) in RPMI 1640 medium containing 10\% FBS supplemented with $10 \%$ penicillin-streptomycin and $1 \%$ insulin-transferrin-selenium A liquid media supplement. Glomeruli were cultured at $37^{\circ} \mathrm{C}$ in a moist $95 \%$ air $/ 5 \% \mathrm{CO}_{2}$ atmosphere.

\section{Incubation of glomeruli with AGEs and blocking with AGEs antibody}

Treatment was performed after 3 days of culture of isolated glomeruli. To block the binding of AGEs to RAGE, $10 \mu \mathrm{g} / \mathrm{mL}$ AGE-pAb or AGE-mAb pre-incubated for 1 hour before $100 \mu \mathrm{g} / \mathrm{mL}$ BSA or AGE-BSA were added for 48 hours. All experiments were repeated two times in each indicated condition.

\section{Double labelling immunofluorescence staining}

The indirect immunofluorescence technique was applied to fixated glomeruli. Double labelling of cultured glomeruli was performed to evaluate the effect of AGE-Ab on AGEs-induced apoptosis and NF-kB p65 expression. In brief, after being incubated with AGEBSA in the absence or pre-treatment of AGE-Ab with a 1 hour interval, glomeruli on glass bottom dish were fixed with $4 \%$ paraformaldehyde for 15 minutes, washed with PBS three times, permeabilized with $2 \%$ Triton X-100 for 5 minutes, blocked with blocking solution (2\% BSA and $2 \%$ FBS in PBS) for 30 minutes, and incubated with primary antibody for 1 hour. For double-label immunostaining of NF-kB p65 expression, anti-NF-kB p65 and anti-nephrin primary antibody were premixed as follows and applied simultaneously. After washing with PBS three times, glomeruli were incubated with fluorescein isothiocyanate (FITC)- and rhodamine-conjugated secondary antibody for 1 hour, then washed again with PBS three times. Sequential double staining was performed to evaluate apoptosis using FITC-conjugated annexin V for 1 hour, blocked with blocking solution for 30 minutes, incubated with anti-nephrin primary antibody for 1 hour, and then incubated with rhodamine-conjugated secondary antibody for 1 hour. The negative control was performed using $2 \% \mathrm{BSA}$ in PBS instead of the primary antibody. 


\section{Quantitative imaging}

Fluorescence images were acquired using confocal laser scanning microscope (Fluoview FV1000, Olympus, Tokyo, Japan) and recorded on a computer using the Olympus Fluoview ver 1.7a viewer. The expression of protein was determined as described previously [19]. Digital pictures of the green and red channels were quantitated using the ImageJ 1.50 software (NIH, MD, USA). Three fields were selected for analysis of each stain. The images were converted to 8-bit grayscale. Then, an outline was drawn around each glomeruli and selected as the region of interest (ROI) to measure area (A) and integrated density (ID) of fluorescence. Next, the mean gray value of background readings (MGV) was measured by selecting five distinct areas in the background with no fluorescence. The corrected optical density $(\mathrm{COD})=\mathrm{ID}-(\mathrm{A} \ddot{\mathrm{O}} \times \mathrm{MGV})$, was calculated. Data were plotted using Prism 6.0 software (GraphPad, San Diego, CA, USA).

\section{Statistical analysis}

All data are presented as mean $\pm \mathrm{SD}$ of two independent experiments. The statistical analysis was performed using the IBM SPSS Statistics 20 software for Windows (IBM Corp., Armonk, NY, USA). Differences between groups were determined by KruskalWallis tests, followed by Mann-Whitney post-tests to calculate statistical significance. Correlation analyses between variables were evaluated using the non-parametric Spearman rank coefficient. Group differences at the level of $p<0.05$ were considered statistically significant.

\section{RESULTS AND DISCUSSION}

The previous study demonstrated that AGEs accumulation resulted in glomerular damage by activating RAGE in podocyte, endothelial cells, mesangial cells, and tubular cells [4]. In this study, we provided in vitro evidences that blocking AGE-RAGE interaction with AGEs antibody protects glomerular cells from apoptosis and NF-kB overexpression induced by AGEs. To the best of our knowledge, this is the first study demonstrating that AGEs antibody protects glomerular damage induced by AGEs. Previously study demonstrated that AGE-RAGE interaction can also be inhibited by pre-incubation of RAGE-Ab. This antibody protects functional and morphological damages of human podocytes induced by peritoneal dialysis fluidand glucose degradation products [20].

\section{AGEs antibody down-regulated AGEs-induced glomerular $N F-\mathbf{k} B$ p 65 overexpression}

To examine the effect of AGEs on NF- $\mathrm{kB}$ p65 expression in glomerular cells, we pre-incubated glomeruli with AGE-pAb or AGE-mAb before AGEBSA exposure. As shown in Figure $1 \mathrm{~A}-\mathrm{B}$, AGEs significantly increased NF- $\mathrm{B}$ p65 expression in cultured glomeruli. However, AGEs-induced over-expression of NF- $\mathrm{BB}$ p65 was significantly inhibited by preincubation of AGE-Ab. AGE-pAb significantly decreased NF-kB p65 expression better than AGE-mAb. Since nephrin expression has been associated with podocyte survival, we studied the effect of AGE-Ab on nephrin expression. The presence of AGE-BSA significantly reduced nephrin expression, thus increased NF-kB/nephrin expression ratio (Figure $1 \mathrm{C}$ ). Both $\mathrm{AGE}-\mathrm{pAb}$ and $\mathrm{CmL}-$ $\mathrm{mAb}$ significantly reduced NF-kB/nephrin expression ratio compared with AGE-BSA-treated group. These findings indicated that AGE-Ab down-regulated AGEsinduced NF-kB p65 over-expression.

AGEs are believed to play a major role in the development of DN. Interaction of AGE-RAGE acti-vates a series of intracellular signaling pathway, inclu-ding NF- $\mathrm{kB}$ transcription factor which initiates and stimulates the production of pro-inflammatory mole-cules that contribute to the pathogenesis of DN [3]. Our study showed that AGE-BSA increased glome-rular NF-kB p65 expression. This finding supported by Bierhaus et al. [21] that binding AGEs to RAGE results in sustained activation of NF- $\mathrm{kB}$ as the result of increased levels of de novo synthesized NF-kB p65. Sustained activation $N F-k B$ is mediated by initial degradation of $\mathrm{I} \mathrm{B}$ protein followed by new synthesis of NF-kB p65 mRNA and protein in the presence of newly synthesized $\mathrm{I}_{k \mathrm{~B}} \alpha$ and $\mathrm{I} \mathrm{BBb}$ [21]. Research conducted by Peng et al. [22] showed that AGEs signal via RAGE generates a signal-specific post-translational modification, or a "barcode" to NF- $\mathrm{KB}$ that mediates a specific gene expression pattern. AGE-RAGE signaling results in NF$\mathrm{kB}$ activation via phosphorylation of NF-kB p65 subunit at T254, S311, S536 residues [22]. AGEs also induces NF- $\mathrm{kB}$ activation mediated by suppression of sirtuin 1 (SIRT1) expression in podo-cytes, which leads acetylation of NF-kB p65 subunit [23].

Unlike many other receptors, the activation of RAGE by AGEs positively upregulates RAGE expression, resulting in sustained RAGE signaling through a NF-kB-dependent mechanism [24,25]. Moreover, activation of NF-kB also upregulates AGEs formation via 
(A)
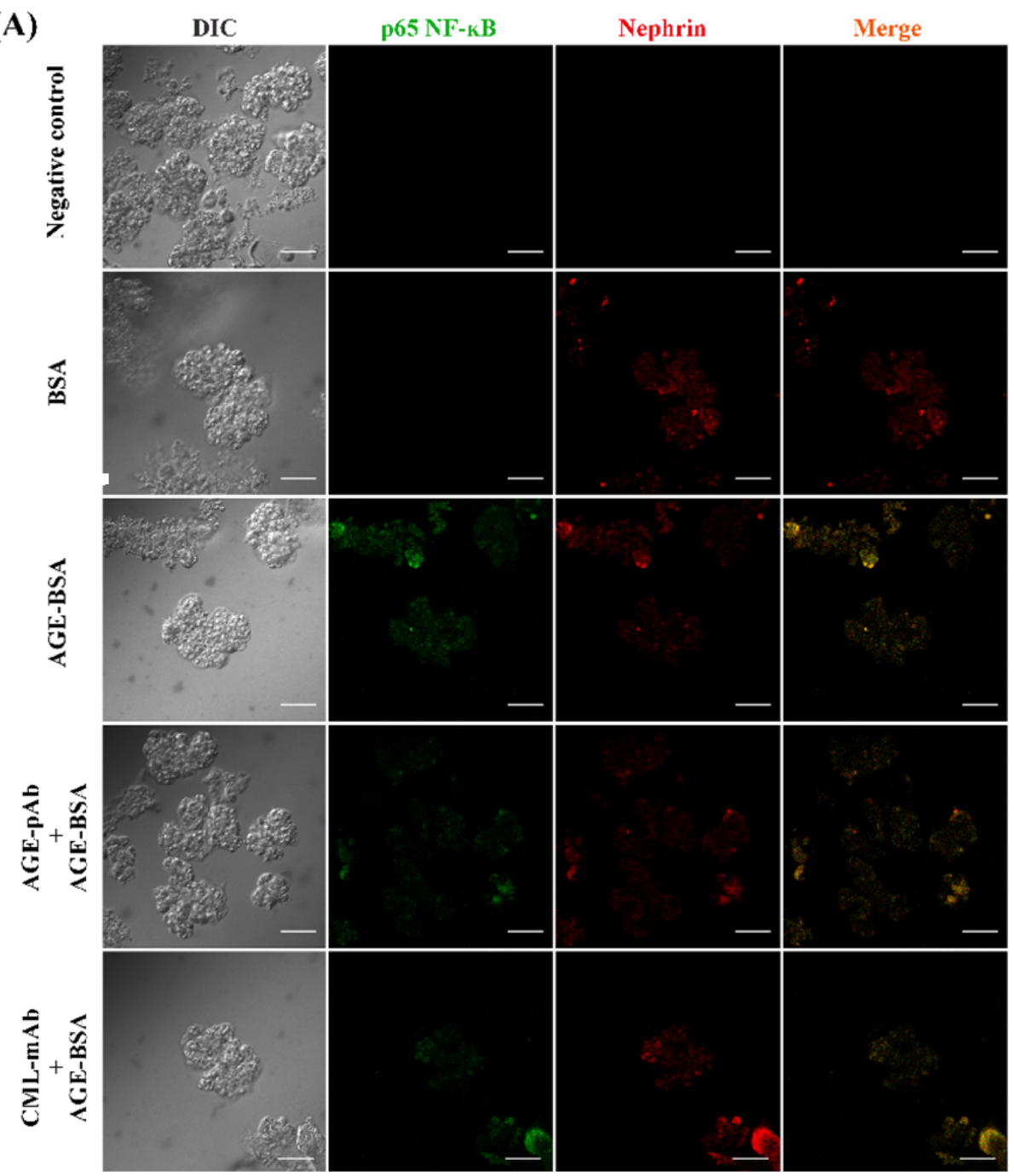

(B)

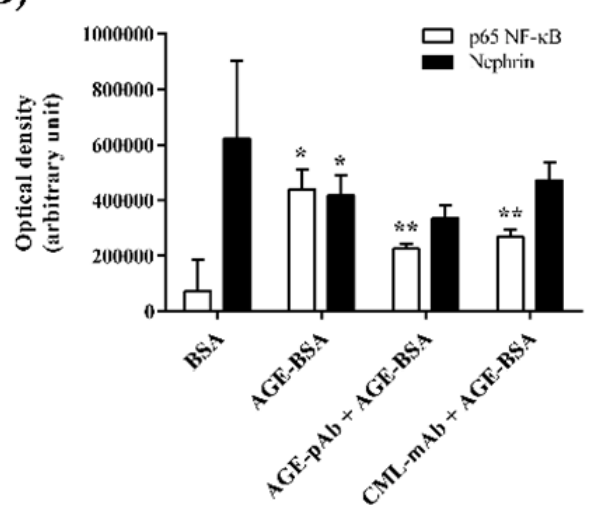

(C)

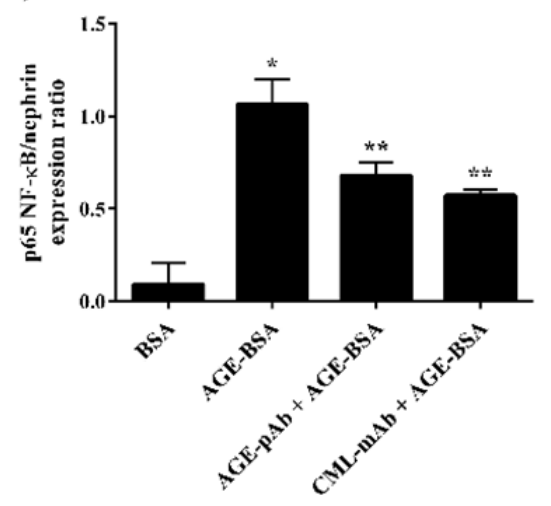

Figure 1. AGEs antibody downregulated glomerular NF- $\mathrm{kB}$ p65 expression induced by AGEs. A. Representative confocal microscopic images of NF-kB p65 (green) and nephrin (red) fluorescence in the glomeruli. BSA was chosen as normal control while AGE-BSA as positive control. The primary antibody was replaced by $2 \%$ BSA in PBS for negative control. Magnification $400 \times$. Scale bars $=50 \mu \mathrm{m}$. B. Bar graphs show the average optical density of NF-kB p65 and nephrin per glomerular. C. Expression ratio of NF-kB p65/nephrin. Data were expressed as mean \pm SD. AGE = advanced glycation end product; $\mathrm{AGE}-\mathrm{pAb}=$ polyclonal anti-AGEs antibody; $\mathrm{BSA}=$ bovine serum albumin; $\mathrm{CmL}-\mathrm{mAb}=$ monoclonal antiNo]-carboxymethyl-lysine antibody; NF-kB = nuclear factor-kappa B. ${ }^{*} \mathrm{p}<0.05$, vs BSA; ${ }^{* *} \mathrm{p}<0.05$, vs AGE-BSA. 


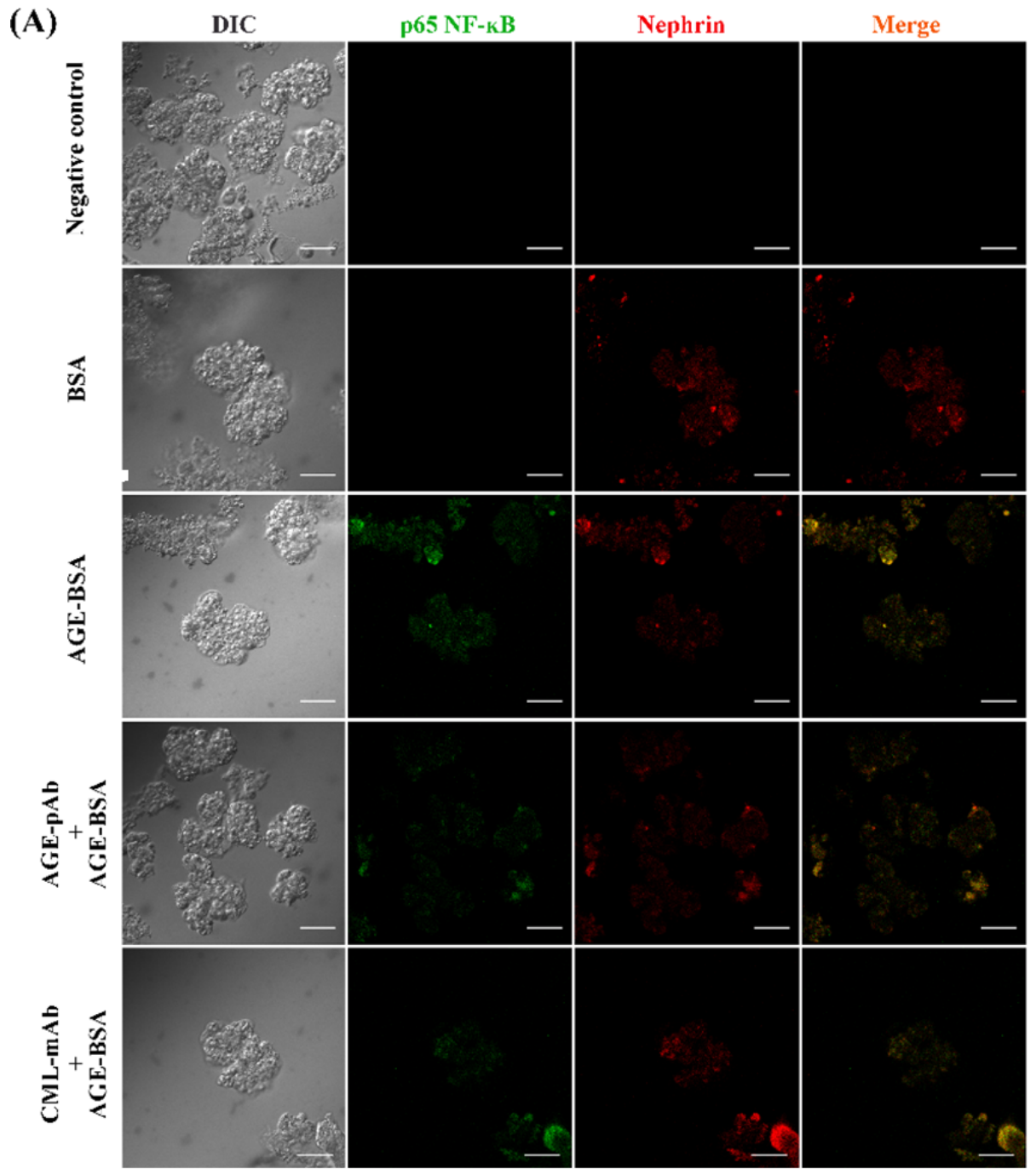

(B)

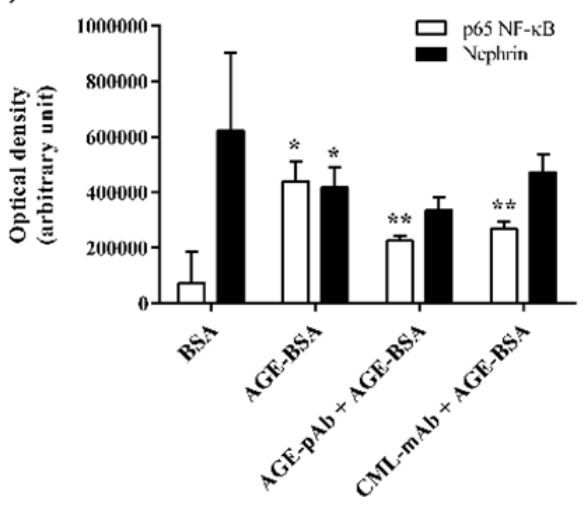

(C)

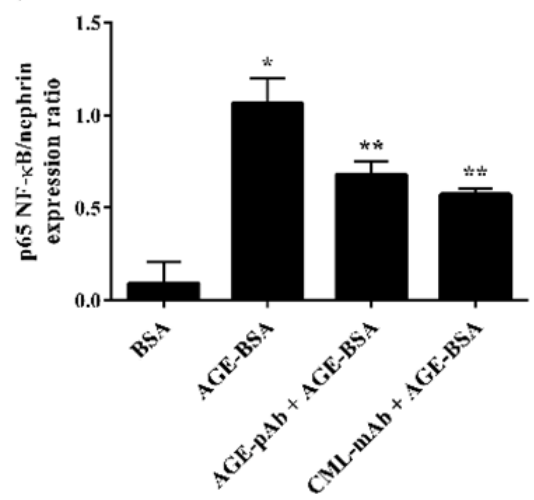

Figure 2. AGEs antibody ameliorated glomerular cells apoptosis induced by AGEs. A. Images show glomeruli in either normal condition or treated with AGE-BSA for 48 hours in the absence or presence of AGE-Ab. Apoptotic cells were stained with FITC-conjugated Annexin V (shown in green) due to phosphatidylserine externalization and nephrin (shown in red). Magnification $400 \times$. Scale bars $=50 \mu \mathrm{m}$. B. Optical density of annexin V and nephrin was calculated using ImageJ. C. Expression ratio of annexin V/nephrin. Data were expressed as mean $\pm \mathrm{SD}$. AGE $=$ advanced glycation end product; $\mathrm{AGE}-\mathrm{pAb}=$ polyclonal anti-AGEs antibody; $\mathrm{BSA}=$ bovine serum albumin; $\mathrm{CmL}-\mathrm{mAb}=$ monoclonal anti-N? carboxymethyl-lysine antibody. ${ }^{*} \mathrm{p}<0.05$, vs BSA; ${ }^{* *} \mathrm{p}<0.05$, vs AGE-BSA. 
suppressing the expression of glyoxalase which inactivates the AGE precursor methylglyoxal [25]. RAGE activation in mesangial cells increases angiotensin-II (Ang-II) production then activates trans-forming growth factor-b-Smad signaling that induces mesangial cell hypertrophy [26]. In addition, Ang-II induces podocyte injury and nephrin inactivation (dephosphorylation) through caveolin-1-dependent mechanism and C-terminal-Src kinase- C-terminal-binding proteinFyn axis $[27,28]$. Reduction of nephrin surface expression is also induced by activation of protein kinase Cmediated nephrin endocytosis [29]. Nephrin deficiency activates NF- $\mathrm{kB}$ and disrupts clustering of membrane raft micro-domains to prevent nephrin-mediated signaling, which affects the podo-cytes or other glomerular cells that interact with podo-cytes [30,31].

\section{AGEs antibody ameliorated AGEs-induced apoptosis of glomerular cells}

Figure 2A-B illustrate the effect of AGEs and AGE$\mathrm{Ab}$ on apoptosis. AGE-BSA treatment resulted in a significant increase of glomerular cells apoptosis and decrease of podocyte nephrin which significantly inhibited by pre-incubation of AGE-Ab. Previously study showed that AGE-BSA induces apoptosis by enhancing FOXO4 binding to a forkhead binding ele-ment in the promoter of Bcl2lll and increasing the acetylation of FOXO4 at lysine residues mediated by downregulation of sirtuin 1 (SIRT1) in cultured podocytes and in glomeruli of diabetic patients [32]. Likewise, AGE-Ab significantly reduced apoptosis/ nephrin expression ratio compared with AGE-BSA-treated group (Figure 2C). Interestingly, we found that nephrin expression significantly preserved in the group of glomeruli under AGE-mAb-treated group better than AGE-pAb-treated group. These results suggested that AGE-Ab had a protective activity on AGEs-induced glomerular cells apoptosis and nephrin deple-tion.

\section{The correlation between NF-kB p65 expression and apoptosis}

A positive correlation was observed between NF- $\mathrm{kB}$ p65 expression and apoptosis $(r=0.818)$ as well as between NF-kB/nephrin and apoptosis/nephrin ex-pression ratio $(r=0.828)$. Taken together, these data provided indirect evidence that AGE-Ab-mediated downregulation of NF-kB p65 expression may contri-bute to inhibition of glomerular cells apoptosis. The results of the present study support the protective role of AGE$\mathrm{Ab}$ against glomerular cells apoptosis, NF-kB p65 overexpression and nephrin depletion induced by AGEs. In this study, we found that polyclonal anti-AGEs antibody protects apoptosis and NF-kB p65 overexpression better than monoclonal anti-CmL antibody. We speculate that AGE-IC formation contributes to these effects. According to Mera et al. [13], we hypothesize that polyclonal AGEs antibody recognizes multiple epitopes on AGE-BSA, thus increases the formation of AGE-IC which induces macrophages-mediated AGE-BSA phagocytosis [13]. However, activated macrophages can downregulate nephrin expression [33]. In this study, monoclonal CmL-specific antibody preserves nephrin expression. $\mathrm{CmL}$ is one of the major immunogen of AGEs that accumulates in all renal compartment of diabetic patient. CmL increases expression of ZEB2 by NF- $\mathrm{kB}$ activation and results in epithelialmesenchymal trans-formation of podocyte which is believed to play a vital role in podocyte depletion and the pathogenesis of albuminuria during DN [34]. Our results showing only minor reduction of nephrin expression on pre-incubation of polyclonal anti-AGEs antibody. The monoclonal anti-CmL antibody obviously exerts pro-tective effect to podocyte with an aim to preserve nephrin expression. Previously study reported that both polyclonal and monoclonal anti-CmL antibody have a significant reactivity to $\mathrm{CmL}$-proteins. More-over, this monoclonal antibody significantly reacted with AGE-BSA as well as BSA modified by several aldehydes such as glyoxal [35].

\section{CONCLUSION}

In conclusion, the present data confirm that pre-incubation of AGEs antibody inhibits apoptosis and $\mathrm{NFkB}$ p65 overexpression in glomeruli exposed to AGEs. Our study demonstrates for the first time that the antibody against AGEs may prevent AGEs-induced glomerular damage.

\section{ACKNOWLEDGMENT}

The authors gratefully acknowledge the Central Laboratory of Life Sciences and the Laboratory of Bioscience, Brawijaya University, Malang, for providing the laboratory facilities.

\section{REFERENCES}

1. Guariguata L, Whiting DR, Hambleton I et al (2014) Global estimates of diabetes prevalence for 2013 and projections for 2035. Diabetes Res Clin Pract. 103 (2): 137-149.

2. Ott C, Jacobs K, Haucke E et al (2014) Role of advanced glycation end products in cellular signaling. Redox Biol. 2:411-429. 
3. Yamagishi SI, Matsui T (2010) Advanced glycation end products, oxidative stress and diabetic nephropathy. Oxid Med Cell Longev. 3 (2): 101-108.

4. Daroux M, Prévost G, Maillard-lefebvre H, Gaxatte C (2010) Advanced glycation end-products : Implications for diabetic and non-diabetic nephropathies. Diabetes Metab. 36: $1-10$.

5. Miyata $T$, Dan $T$ (2008) Inhibition of advanced glycation end products (AGEs): An implicit goal in clinical medicine for the treatment of diabetic nephropathy? Diabetes Res Clin Pract. 825: 25-29.

6. Pashikanti S, Alba DR De, Boissonneault GA, Cervanteslaurean D (2010) Free Radical Biology \& Medicine Rutin metabolites: Novel inhibitors of nonoxidative advanced glycation end products. Free Radic Biol Med. 48 (5): 656663.

7. Joglekar MM, Panaskar SN, Arvindekar AU (2013) Inhibition of advanced glycation end product formation by cymene - A common food constituent. J Funct Foods. 6: $107-115$.

8. Losso JN, Bawadi HA, Chintalapati M (2011) Inhibition of the formation of advanced glycation end products by thymoquinone. Food Chem. 128 (1): 55-61.

9. Sang H, Gu J, Yuan J, Zhang M (2014) The protective effect of smilax glabra extract on advanced glycation end products-induced endothelial dysfunction in HUVECs via RAGE-ERK1/2-NF-KB pathway. J Ethnopharmacol. 155(1):785-795

10. Feng L, Zhu M, Zhang $M$ et al (2013) Protection of glycyrrhizic acid against AGEs-induced endothelial dysfunction through inhibiting RAGE/NF- $\kappa \mathrm{B}$ pathway activation in human umbilical vein endothelial cells. J Ethnopharmacol. 148 (1): 27-36.

11. Reddy VP, Beyaz A (2006) Inhibitors of the Maillard reaction and AGE breakers as therapeutics for multiple diseases. Drug Discov Today. 11: 646-654.

12. Mashitah MW, Azizah N, Samsu N et al (2015) Immunization of AGE-modified albumin inhibits diabetic nephropathy progression in diabetic mice. Diabetes, Metab Syndr Obes Targets Ther. 8: 347-355.

13. Mera K, Nagai R, Takeo K et al (2011) An autoantibody against $\mathrm{N}$-(carboxyethyl) lysine (CEL): Possible involvement in the removal of CEL-modified proteins by macrophages. Biochem Biophys Res Commun. 407 (2): 420-425.

14. Turk Z, Ljubic S, Turk N, Bojan B (2001) Detection of autoantibodies against advanced glycation endproducts and AGE-immune complexes in serum of patients with diabetes mellitus. Clin Chim Acta. 303: 105-115.

15. Baydanoff S, Konova E, Ivanova (1996) Determination of anti-AGE antibodies in human serum. Glycoconj J.
13:335-339.

16. Katsuya K, Yaoita E, Yoshida Y et al (2006) An improved method for primary culture of rat podocytes. Int Soc Nephrol. 69:2101-2106.

17. Takemoto M, Asker N, Gerhardt $\mathrm{H}$ et al (2002) Technical Advance A New Method for Large Scale Isolation of Kidney Glomeruli from Mice. Am J Pathol. 161 (3): 799805.

18. Liu X, Fan Q, Yang G et al (2013) Isolating glomeruli from mice: A practical approach for beginners. Exp Ther Med. 5: 1322-1326.

19. Mallipattu SK, Liu R, Zheng F et al (2012) Krüppel-like factor 15 (KLF15) is a key regulator of podocyte differentiation. J Biol Chem. 287(23):19122-19135.

20. Müller-Krebs S, Kihm LP, Madhusudhan T et al (2012) Human RAGE antibody protects against AGE-mediated podocyte dysfunction. Nephrol Dial Transplant. 27 (8): 3129-3136.

21. Bierhaus A, Schiekofer S, Schwaninger M et al (2001) Diabetes-associated sustained activation of the transcription factor nuclear factor-kappaB. Diabetes. 50(12):2792-2808.

22. Peng Y, Kim J, Park $H$ et al (2016) AGE-RAGE signal generates a specific NF- $\kappa$ B RelA "barcode " that directs collagen I expression. Nat Publ Gr. 1-10.

23. Liu R, Zhong Y, Li X et al (2014) Role of transcription factor acetylation in diabetic kidney disease. Diabetes. 63 (7): 2440-2453.

24. Fritz G (2011) RAGE: a single receptor fits multiple ligands. Trends Biochem Sci. 36 (12): 625-632.

25. Yao D, Brownlee M (2010) Hyperglycemia-induced reactive oxygen species increase expression of the receptor for advanced glycation end products (RAGE) and RAGE ligands. Diabetes. 59: 249-255.

26. Fukami K, Ueda S, Yamagishi S et al (2004) AGEs activate mesangial TGF-beta-Smad signaling via an angiotensin II type I receptor interaction. Kidney Int. 66 (6): 2137-2147.

27. Ren Z, Liang W, Chen C et al (2012) Angiotensin II induces nephrin dephosphorylation and podocyte injury: role of caveolin-1. Cell Signal. 24 (2): 443-450.

28. Yu L, Lin Q, Feng et al (2013) Inhibition of nephrin activation by c-mip through Csk-Cbp-Fyn axis plays a critical role in Angiotensin II-induced podocyte damage. Cell Signal. 25 (3): 581-588.

29. Tossidou I, Teng B, Menne J et al (2010) Podocytic PKCalpha is regulated in murine and human diabetes and mediates nephrin endocytosis. PLoS One. 5 (4): e10185.

30. Li X, Chuang PY, D'Agati VD et al (2015) Nephrin Preserves Podocyte Viability and Glomerular Structure and Function in Adult Kidneys. J Am Soc Nephrol. 1-17. 
31. Hussain S, Romio L, Saleem M et al (2009) Nephrin Deficiency Activates NF-KB and Promotes Glomerular Injury. J Am Soc Nephrol. 20 (8): 1733-43.

32. Chuang PY, Dai Y, Liu R et al (2010) Alteration of forkhead box $\mathrm{O}$ (foxo4) acetylation mediates apoptosis of podocytes in diabetes mellitus. PLoS One. 6 (8): e23566.

33. Ikezumi Y, Suzuki T, Karasawa T et al (2008) Activated macrophages down-regulate podocyte nephrin and podocin expression via stress-activated protein kinases. Biochem Biophys Res Commun. 376 (4): 706-711.

34. umar PA, Welsh GI, Raghu G et al (2016) Carboxymethyl lysine induces EMT in podocytes through transcription factor ZEB2: Implications for podocyte depletion and proteinuria in diabetes mellitus. Arch Biochem Biophys. 590: 10-19.

35. Koito W, Araki T, Horiuchi S, Nagai R (2004) Conventional Antibody against NE-(Carboxymethyl) Lysine (CmL) Shows Cross-Reaction to $N \mathcal{E}$ (Carboxyethyl)Lysine (CEL): Immunochemical Quantification of $\mathrm{CmL}$ with a Specific Antibody. J Biochem. 136 (6): 831-837. 